Published as: Ooms, K., Andrienko, G., Andrienko, N., De Maeyer, P. \& Fack, V. (2012), Analysing the spatial dimension of eye movement date using a visual analytic approach, Expert Systems with Aplications, vol. 39 (1), p. 1324-1332.

\title{
Analysing the spatial dimension of eye movement data using a visual analytic approach
}

\author{
$\underline{\text { Kristien Ooms }}^{\mathrm{a}}$, Gennady Andrienko ${ }^{\mathrm{b}}$, Natalia Andrienko ${ }^{\mathrm{b}}$, Philippe De Maeyer ${ }^{\mathrm{a}}$, Veerle \\ Fack $^{\mathbf{c}}$ \\ ${ }^{a}$ Ghent University,Department of Geography, Ghent University, Krijgslaan 281 S8, B-9000 \\ Ghent, Belgium, \{Kristien.Ooms; Philippe.DeMaeyer $\} @ U G e n t . b e$ \\ TEL: +32 (0)9 2644636 ; FAX: +32 (0)9 2644985 \\ ${ }^{\mathrm{b}}$ Fraunhofer Institute, for Intelligent Analysis and Information Systems (IAIS), Schloss \\ Birlinghoven, 53754 Sankt Augustin, Germany, \{ Gennady.Andrienko; Natalia.Andrienko \\ \} @ IAIS.Fraunhofer.de \\ ${ }^{\mathrm{c}}$ Ghent University, Department of Applied Mathematics and Computer Science, Ghent \\ University, Krijgslaan 281 S9, B-9000, Ghent, Belgium, Veerle.Fack@UGent.be
}

\begin{abstract}
Conventional analyses on eye movement data only take into account eye movement metrics, such as the number or the duration of fixations and length of the scanpaths, on which statistical analysis is performed for detecting significant differences. However, the spatial dimension in the eye movements is neglected, which is an essential element when investigating the design of maps. The study described in this paper uses a visual analytics software package, the Visual Analytics Toolkit, to analyze the eye movement data. Selection, simplification and aggregation functions are applied to filter out meaningful subsets of the data to be able to recognize structures in the movement data. Visualising and analysing these patterns provides essential insights in the user's search strategies while working on a(n interactive) map.
\end{abstract}

Keywords: map design; visual analytics; eye movements; mental map 


\section{Introduction}

In the field of Human Computer Interaction (HCI), usability studies are conducted throughout a product's design lifecycle to improve its design and functionalities towards the end user. Many types, structures, and techniques exist among these usability studies. The selection of the 'best' method to tackle a certain problem or question depends on a number of factors: stage in the product's development, qualitative or quantitative output, type of (research) question, among others. (Nielsen, 1993; Rubin \& Chisnell, 2008) ISO 9245-11, a well known standard in the field of User Centred Design (UCD), describes usability in terms quality of use or the efficiency, effectiveness and satisfaction with which a user achieves a certain goal on a specific system, such as a map (Bevan, 1995; ISO, 1994).

The goal of a good map design is to present the information towards the user in an effective way: the user has to be able to interpret the (spatial) information correctly, but also efficiently. This latter element is related to how the user interprets, processes, and stores 'internally' the information presented to him. In order to be able to improve the design of a map towards the user, it is thus essential to obtain insights in this cognitive or mental map. (Downs \& Stea, 1977; Montello, 2002)

From the long list of possible usability evaluation methods, the eye tracking technique is considered to be the most suitable to obtain these insights in an objective way. Furthermore, throughout the entire study the user's Point of Regard (POR) is registered, which is the location where he was looking on the screen at a certain timestamp. (Duchowski, 2007; Rayner, 1998) Most usability studies focus on quantitative parameters: number of fixations, duration of the fixations, length of saccades, among others (Jacob \& Karn, 2003; Poole \& Ball, 2006). However, these parameters do not take into account the spatial context of the data, which is essential when dealing with maps and their design. Moreover, most software packages accompanying the eye tracking devices are not fully suitable to study this spatial dimension of the eye movement data. Consequently, a more qualitative and visual analytic approach is needed to be able to detect patterns in the user's behaviour, and thus in his scanpaths while working with these maps. In a number of recent studies the eye tracking method has been used to study the design of maps and their usability: their symbology (Brodersen, Andersen, \& Weber, 2001), the map animation (Fabrikant, Rebich-Hespanha, Andrienko, Andrienko, \& Montello, 2008) and the design of the map interface (Coltekin, Heil, Garlandini, \& Fabrikant, 2009).

But eye movement data show similarities with other kinds of movement data: long list of locations $(x, y)$ at a certain timestamp $t$. A number of software packages exist which are able to visualise and analyse this type of movement data and which may thus also be able to handle the eye movement data or the scanpaths as well. The Visual Analytics Toolkit (also known as CommonGIS) is such a software package. Its suitability to visualise the eye movement data in a meaningful way is already briefly demonstrated in the work of Fabrikant et al. (2008). The Visual Analytics Toolkit is developed at the Fraunhofer Institute IAIS (Sankt Augustin, Germany). Its functionalities - including the aggregation and clustering of the movement data are described in a number of articles: e.g. Andrienko et al. (2007), Andrienko and Andrienko (2010). 
The goal of this paper is thus to get insights in the user's cognitive processes while working with dynamic and interactive maps using the eye tracking technique. Spatial patterns in the user's search strategies on these maps hold information on how users process, store and analyze the information presented to them. The Visual Analytics Toolkit is used to select, summarize and aggregate the massive amount of eye movement data obtained during the user study. The design of this study is described in Section 2. The visual analyses are described and discussed in Section 3. A conclusion related to these results is presented in Section 4.

\section{Study design}

The tests were conducted in the Eye Tracking laboratory of the Department of Experimental Psychology at Ghent University, Belgium. This laboratory is equipped with an Eye Link 1000 device from SR Research (Mississauga, Ontario, Canada) and samples a person's POR at a rate of $1,000 \mathrm{~Hz}$ (or once every $\mathrm{ms}$ ). The movements from one eye only are recorded during the tests. The recorded eye movements of 14 subjects were analyzed. All participants were students and most of them studied courses at the Faculty of Psychology and Educational Sciences, Ghent University.

During the tests, the participants were asked to locate five names on a map, which were listed on the right side of the actual map. As a consequence, the participant had to perform a visual search on the map. This is a operation which is done frequently when a user employs a map: he is trying to find the location of a certain object on the map (such as a city, landmark, crossroad). The user has to orientate and interpret the map content and subsequently search for the location of the target object. The task the users had to execute during this study thus allows studying patterns in the search behaviour which might give insights in how users orientate, process and store the (spatial) information on the map.

Each demo-map had the same simple background with point objects (symbolizing cities) and associated name labels. After 50s the map image was translated horizontally over a fixed difference, simulating a pan operation. This simulation has a duration of one second. The list with the five names had also changed during this translation: two new names are displayed and three which were already in the former list (but on a different location in the list). Again the user had to locate these five names in the map. It was decided to display two different lists of five names to make sure that the user could not track the relative position of the labels in the list during the transition. Since the list is visible during the entire trial, the user did not had to remember all five names reducing the mental workload. The duration of the display of the initial view (50s) is based on reaction time measurement conducted earlier. In total, twenty demo-maps were displayed to the participants in a random order. An example of such a demo-map is depicted in Figure 1.

During the tests, the eye movements of the participants were recorded. Furthermore, the participants were asked to indicate when they found a label by pushing a button. To avoid distracting the users too much from their main task (the visual search) they had to push the same button for each label. Otherwise they might look down to the joystick to look for the button they had to push for a specific label, which would disturb both the time measurements and the recordings from the eye tracker. The combination of the time measurements form the button actions and the location where the user was looking (derived from the eye movement recordings) allows identifying if and which label was found. 


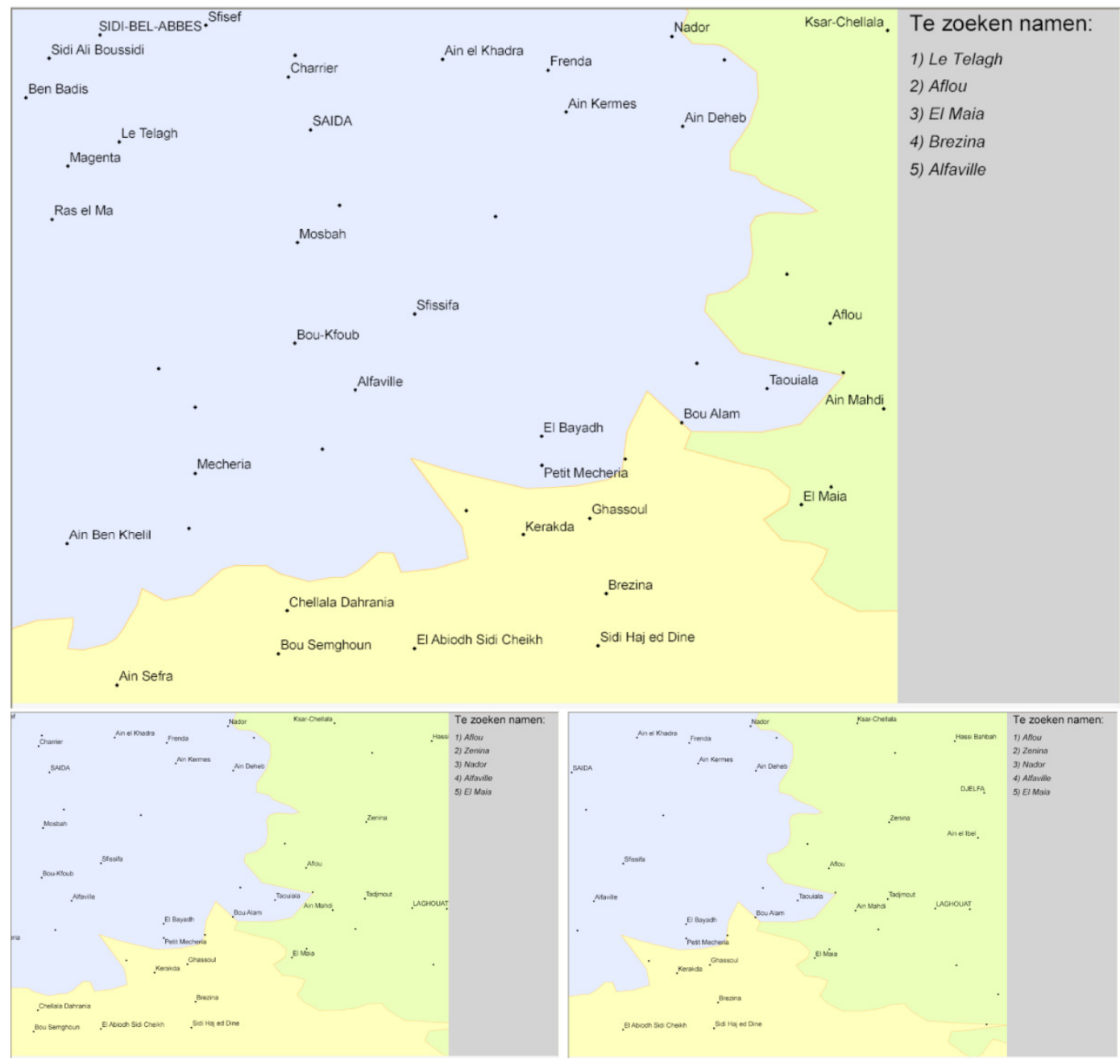

Figure 1: Example of a demo map with on top the initial view, bottom left a view during the panoperation and bottom right the final view

The obtained eye movement data is imported into The Visual Analytics Toolkit, where the eye movements (scanpaths) are treated as if it were trajectories from for example GPS tracks. In the Visual Analytics Toolkit each point corresponds to a stop and thus to a fixation. The saccades are visualised as lines between the corresponding fixations. In total 77069 points were loaded into the Toolkit, based on which the trajectories or scanpaths are visualised. On average, 344 points or fixations are registered per trial, with a minimum of 123 and a maximum of 719 . The average number of fixations per user over all trials is 5505 . 


\section{Results \& Discussion}

Since eye movement studies result in a vast amount of data, the obtained view is totally overcrowded when visualising the movement data of all participants, on all demo-maps and during the whole time interval, even when the scanpaths are visualised with a transparency of $50 \%$. This is depicted in Figure 2. Consequently, it is not possible to detect any patterns and draw any conclusions from this dataset as a whole. Filtering techniques based on some parameters are needed to select only a meaning subset of the scanpaths. The possibilities of the filtering techniques in The Visual Analytics Toolkit and their results are described in the Section 3.1 .

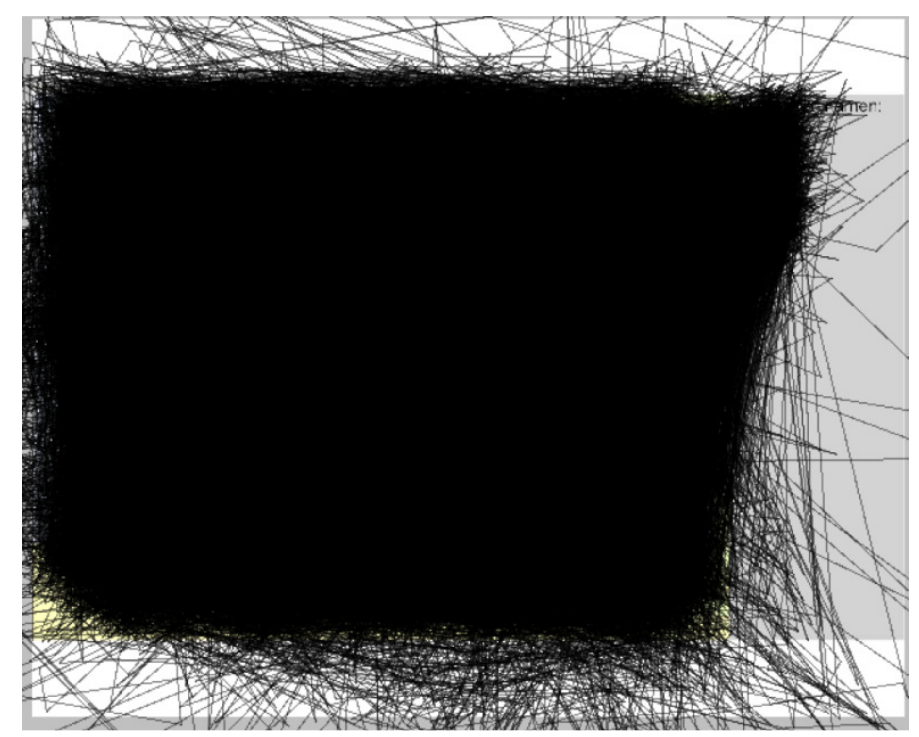

Figure 2: Overcrowded visualisation if all eye movements are selected

\subsection{Filtering of the movement data}

The parameters on which the data can be filtered are linked with the scanpaths attributes on the one hand or with a time interval on the other hand. In Figure 3, filtering techniques on both types of parameters are depicted and the scanpaths are visualised with a transparency of 50\%. Figure $3 a$ presents all scanpaths of one participant only, on all demo-maps. Figure $3 b$ depicts the scanpaths of all participants for all demo-maps but only during the first ten seconds the each trial. It is clear that more restricted selection techniques are needed to be able to detect any patterns in the movement data: shorter time intervals and/or a combination of several filtering parameters. 


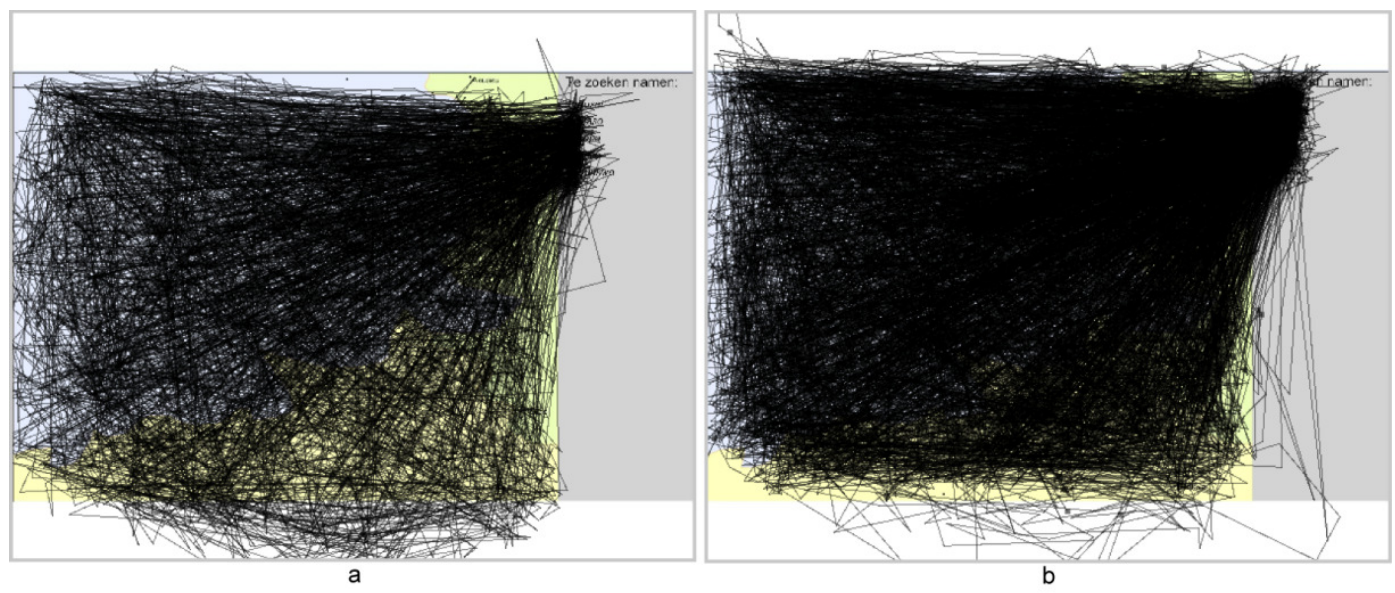

Figure 3: Overcrowded visualisation when minor selections are applied (a. one participant, b. time interval [0s-10s])

In Figure 4, a time series of the movement data is presented with subsequent time intervals of 500ms. This time series allows visualising and analyzing the evolution of the scanpaths over time: how do users scan the map and is there a difference in the search pattern before and after the simulated pan operation?

From this time series it can be derived that nearly all users start looking at the map near its centre, probably to get an initial impression of the overall structure. Next, almost all scanpaths are directed towards the list with the five names, which is interpreted during a certain amount of time. After the first second, most users have started searching for the names on the map. By visualising the actual scanpaths it becomes clear that most users start searching for the names in the upper part of the map. After two seconds, the search patterns are more homogeneously spread over the map which still continues before the simulated interaction. This simulation has duration of one second and occurs during the time interval [50,000-51,000]. From the visualised scanpaths it can be derived that during the first half of this interval, the scanpaths are still distributed rather evenly over the entire view. However, during the second part of the interaction, [50,500-51,000ms], the attention of the users is again drawn to the list with the five names. At this moment, the new map display is not in its final position but the list with five names has already changed. Again the search of the names on the map starts in its upper part, but now the scanpaths have a more straight and diagonal direction. Two seconds after the end of the simulation, the search pattern of the users is again more evenly spread across the map's content. 


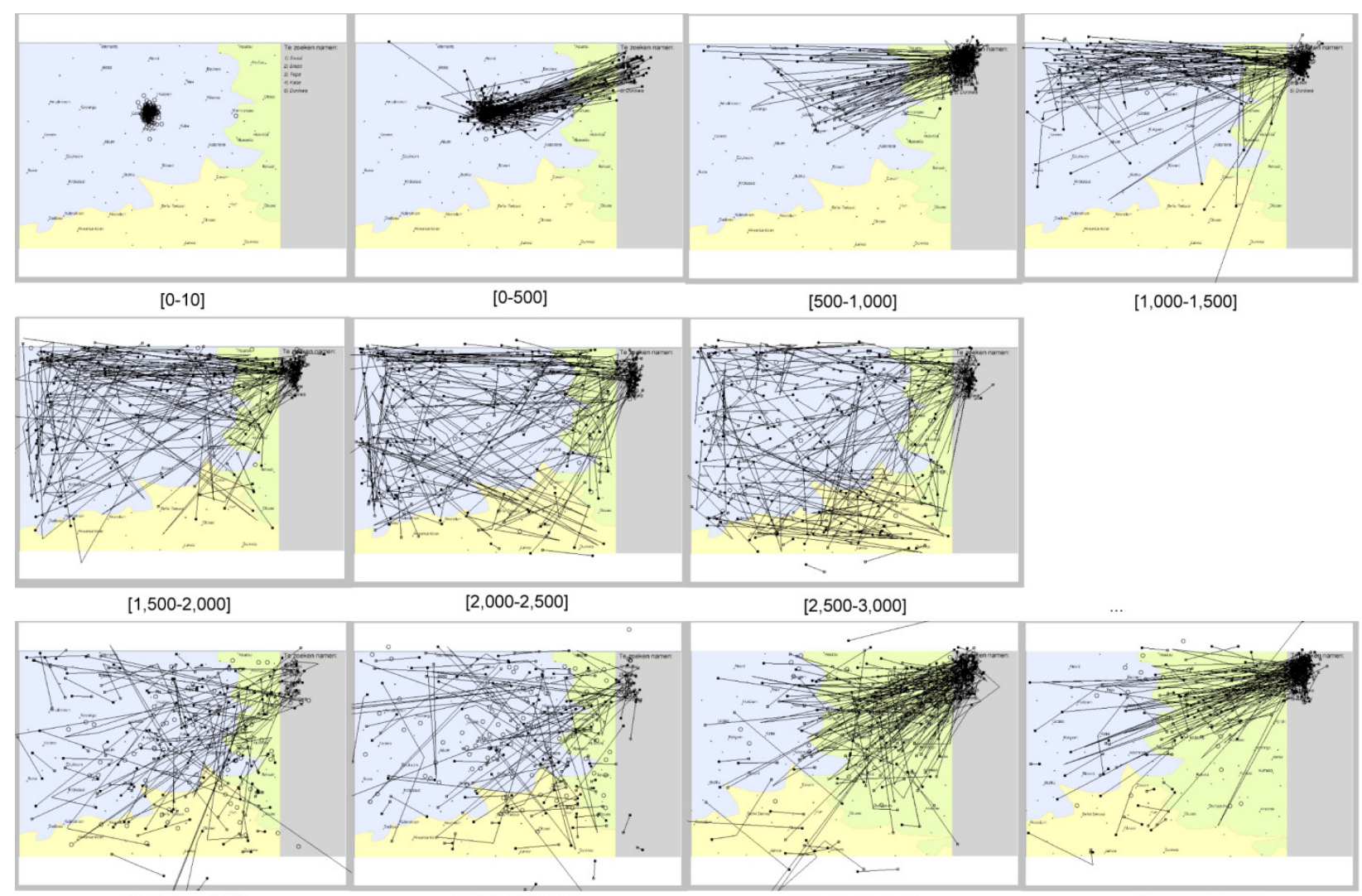

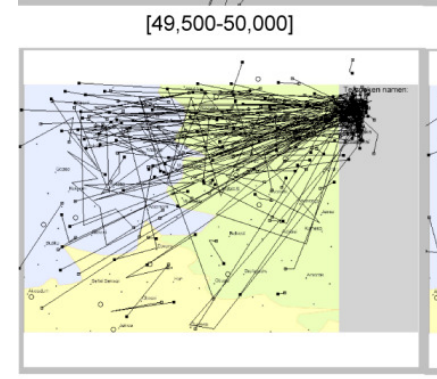

$[51,500-52,000]$

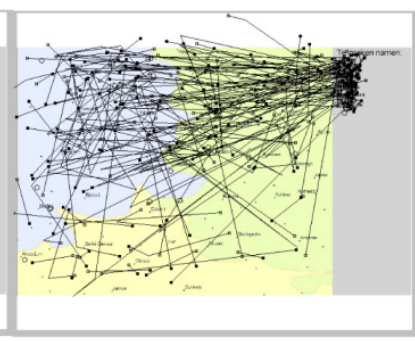

$[52,000-52,500]$

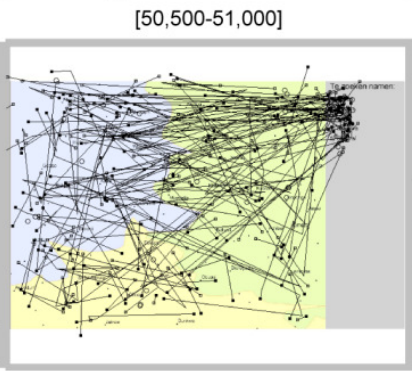

$[52,500-53,000]$

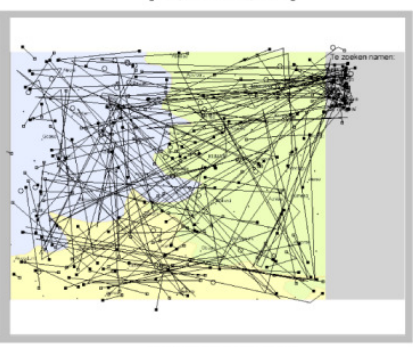

$[53,000-53,500]$

Figure 4: Time series to visualise the evolution of the scanpaths over time

The scanpaths visualised in Figure 5 are a result of the combination of two filtering parameters, namely two attributes: participant (rows) and demo-map (columns). This type of filtering allows detecting if there are any differences in the search behavior of a user when a different map - and thus a different layout in labels - was presented on the one hand, but also if there are differences in the search behavior of different users on a same map. Person 1, for example, does not look very often in the list with names on the right, whereas Person 2 and Person 3 check this list more frequently. The search patterns of Person 2 are very straight whereas these of Person 1 appear to be more chaotic and rather long. The background of the maps (the distribution of the labels) also has an influence on the scanpaths, but this is less clear than the personal differences mentioned before. Map 2 has for example a collection of scanpaths going from the list on the top right to the middle of the map on the left whereas on Map 3 this collection is directed towards the lower right and left corner of the map. 


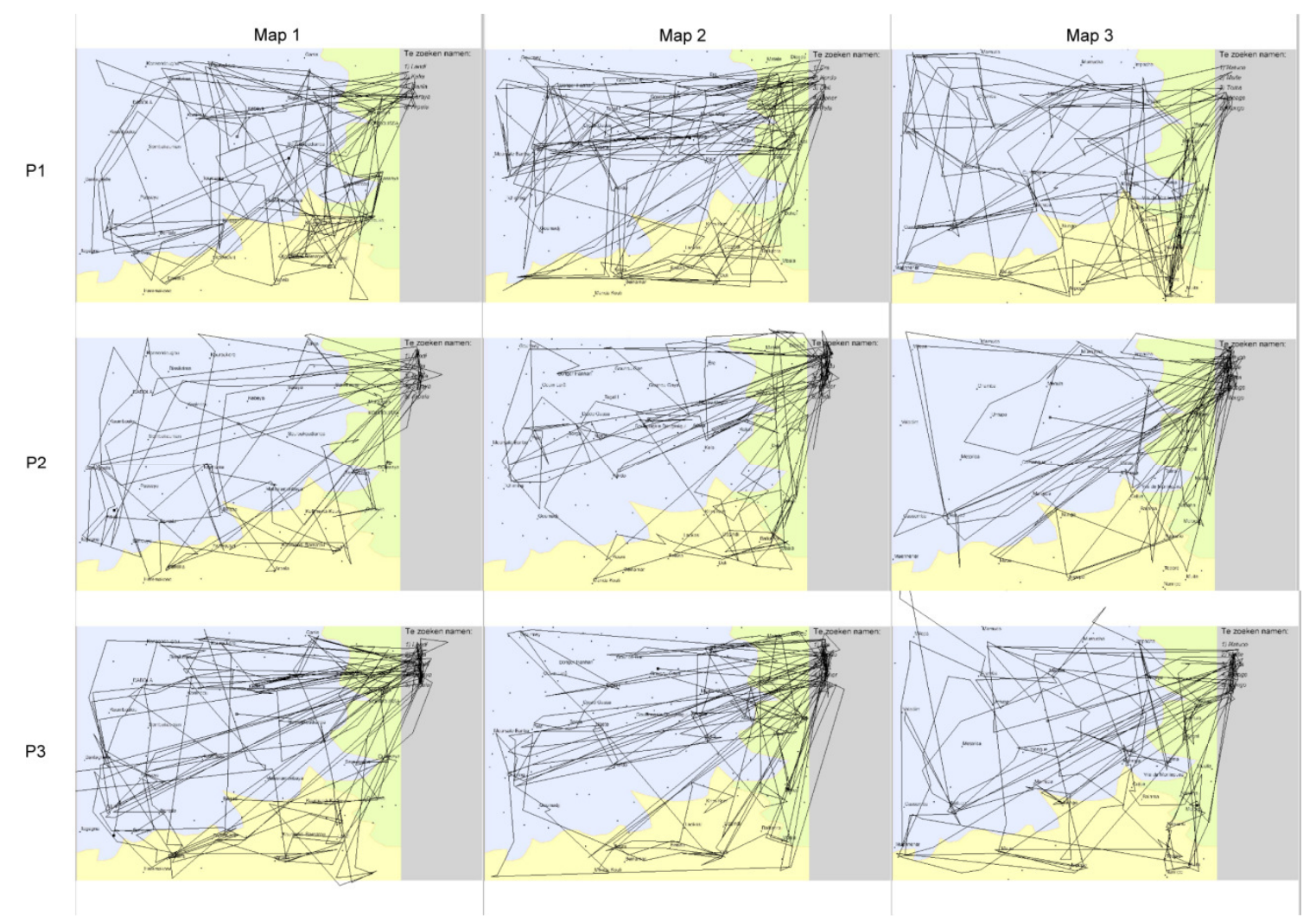

Figure 5: Filtering the scanpaths based on two attributes: participant and demo-map

The scanpaths in the first picture of Figure 6 are only filtered on one attribute: the demo-map which is used. In the two other pictures in Figure 6, the movement data is also filtered on a time interval. The second picture shows all eye movements before the simulated pan-operation whereas in the last picture only the ones after the simulation are visualised. The blue polygons presented along with the scanpaths are the Interest Areas (IAs): the location of the ten labels a user had to locate plus the location of the list with the five names. Some patterns in the movement data are showing when applying this last filtering strategy, but stricter simplification and/or aggregation of this subset of the data is needed to be able to retain only the main structures in the trajectories. These simplification and aggregation techniques are described in more detail in Section 3.2.
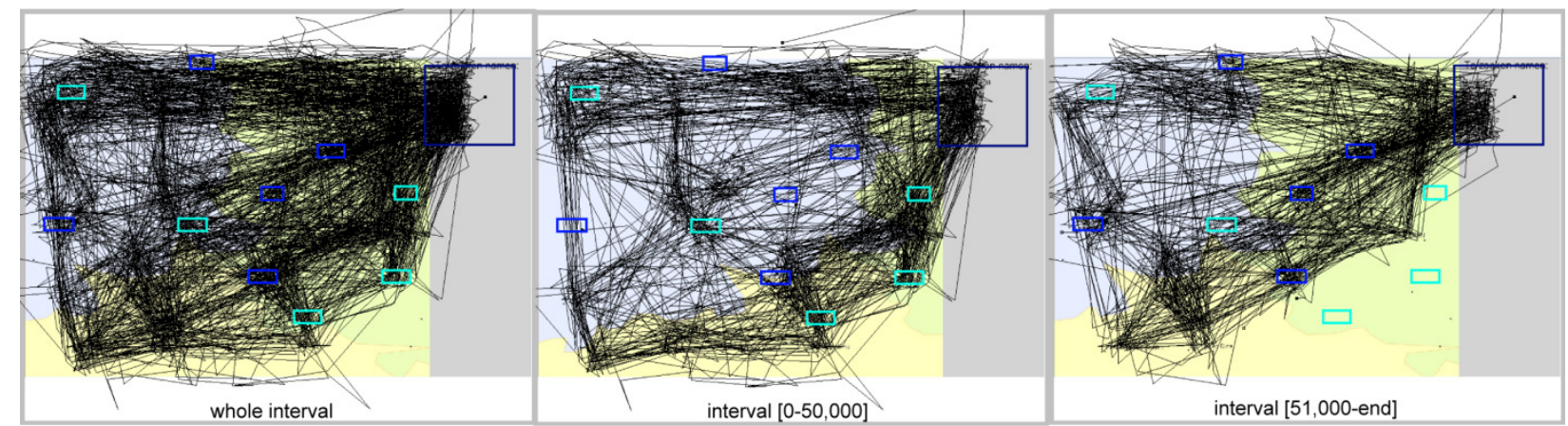

Figure 6: Filtering the scanpaths based on one demo-map and time interval 


\subsection{Simplification and aggregation of the data}

As indicated before, an eye tracking experiment quickly results in a vast amount of data from which no meaningful patterns of conclusions can be derived as such. The patterns are hidden in the somewhat chaotic representation of all scanpaths registered during the experiment. Similar trajectories may be present in the data, but they are concealed by other scanpaths crossing the same area. Aggregating and simplifying the trajectories of the scanpaths is an essential technique to retain only the relevant structures in the data and filter out the minor deviation from the main search strategy. The Visual Analytics Toolkit has a number of options which allow simplifying and aggregating the data with the aid of standard or user defined areas in the view.

One function to aggregate the data in The Visual Analytics Toolkit is called 'Generalise and Summarise Trajectories'. With this function, the area is divided into a set of Voronoi polygons which reflect the density of PORs and minimizes the distortion of the scanpaths. Based on the Voronoi tessellation, the trajectories are divided into individual segments which are subsequently aggregated if they go from and to the same Voronoi polygon pair. A detailed description of this function and its related algorithms can be found in Andrienko and Andrienko (2010).

After some testing it was decided that the following parameter values give the best results regarding the Voronoi tessellation and the clustering of the eye movements:

-Minimal angle of direction change (degree): 10

-Minimum radius around a position (pixels): 30

-Maximum radius around a position (pixels): 60

Different time filters can be applied to the clustered data to visualise only a small subset in an aggregated way. In Figure 7, this clustering technique is applied to the eye movement data of all users on one demo-map. The first picture shows the eye movements before the simulation and the second one the movements after the simulation. Clustering this dataset allows visualising the main movement patterns and thus facilitating its interpretation. In the last picture of Figure 7 the aggregated moves of the scanpaths during the first two seconds only are depicted. This result is in correspondance with the output from the time series discussed earlier in section 3.1.

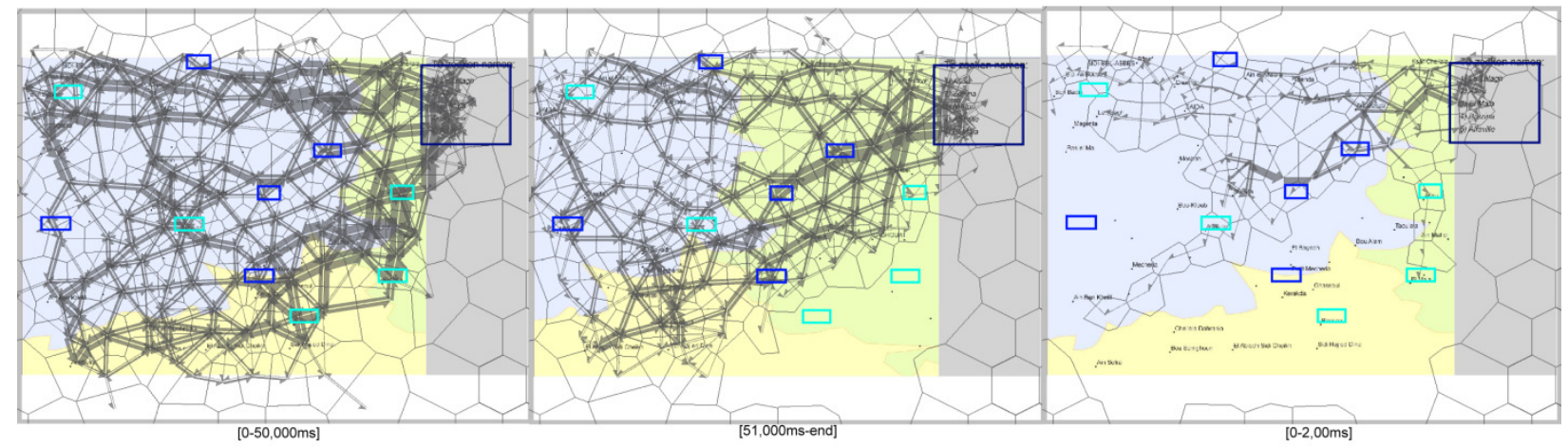

Figure 7: Generalization and summarization of the scanpaths

Furthermore, the data can be simplified and summarized based on a number of user defined areas. Regarding eye movement data, the IAs can be used to investigate the number of scanpaths between these areas. Especially the number of movements between the list with names and the locations of the names is very interesting. These simplified trajectories which are visualised as 
moves between areas can furthermore be aggerated in clusters. In this case, the clustering is not based on the calculated Voronoi tesselation, but on the user defined areas. These simplified and summarized moves between the interest areas are depicted in Figure 8 and Figure 9 respectively, both for before (a.) and after (b.) the simulation, for all users but for one demo-map only.

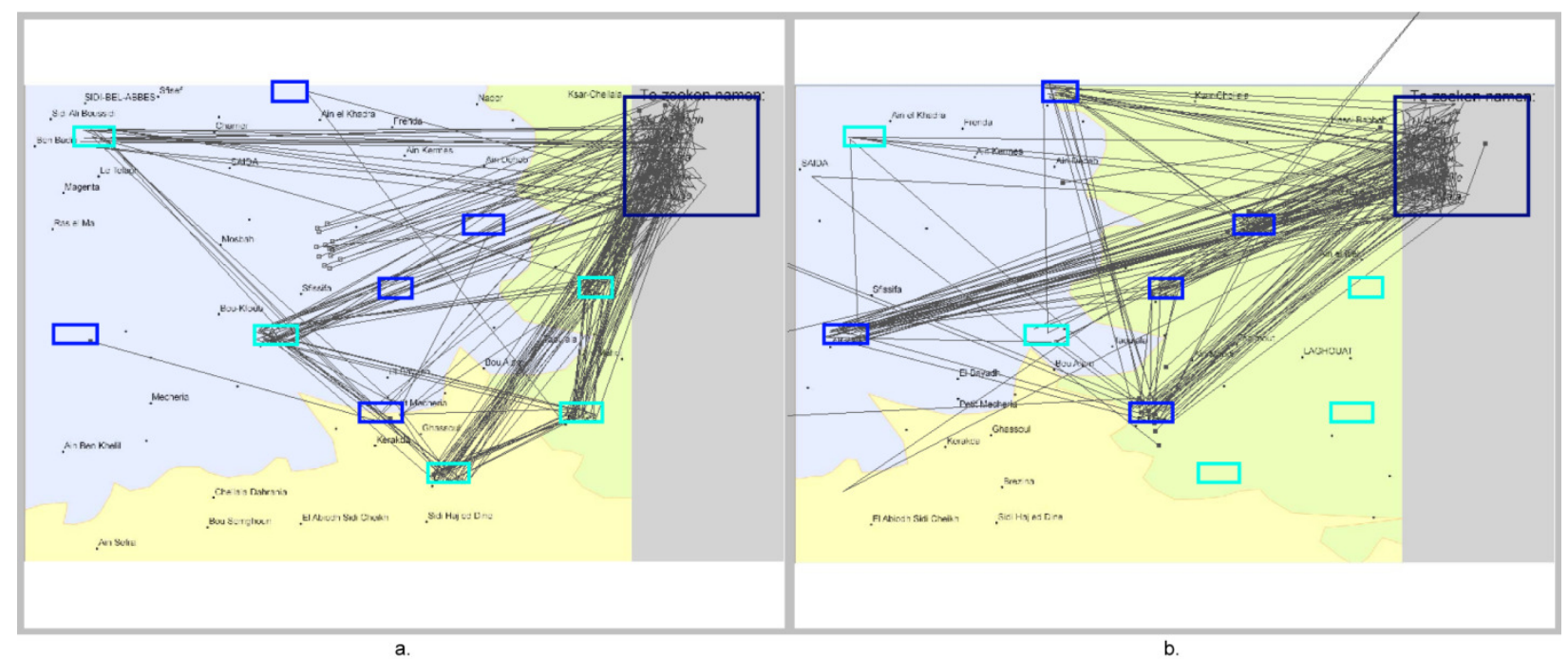

Figure 8: Simplification of the scanpaths based on crossed areas

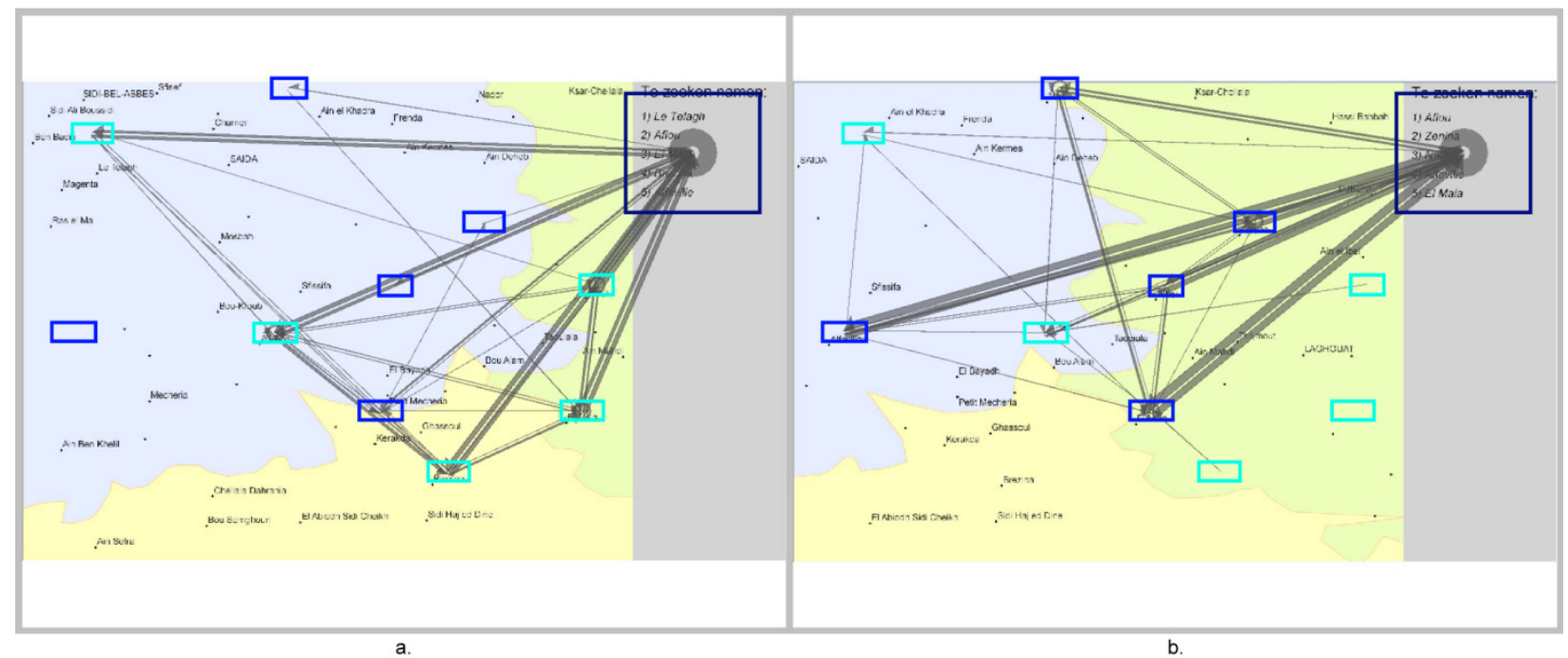

Figure 9: Aggregation of the scanpaths based on crossed areas

Both pictures before the interaction (a.) in Figure 8 and Figure 9 show that the movements are directed towards the light blue rectangles. These rectangles correspond to the locations of the five names which appear in the list before the simulation. The darker blue rectangles correspond to the five labels which have to be located after the interaction. During the first interval, almost no moves are directed towards these latter rectangles. The pictures of the aggregated and simplified movements after the interaction (b.) show the opposite structure. In this case, almost all movements are directed towards the darker rectangle and thus to the locations of the five labels which had to be located after the simulation. 


\subsection{Dividing the trajectories or scanpaths}

The Visual Analytics Toolkit also has the possibility to split up existing trajectories based on (user defined) areas they cross. This is a very interesting tool to filter out the part of the scanpaths between two IAs, visualising the user's search strategy. In this case, the trajectories between when the user looked in the list (which is defined as an IA) and when he found a specific label (also indicated by an IA) can be obtained. The resulting (partial) scanpaths for finding a specific label on a certain map are depicted in Figure 10. In each picture, the scanpaths for finding a specific label on a map for all users are depicted. The rectangles on the map correspond to the locations of the labels on the map which have to be found before (light blue) and after (dark blue rectangles) the simulation. These divided and selected trajectories indicate that some labels are more easily found by the participant than others. Some labels after the simulation are located directly with a straight line between the list and the label: Mia, Bokin and Lankoe. These labels were already present in the list before the interaction $(*)$ and the some users remembered its relative location.
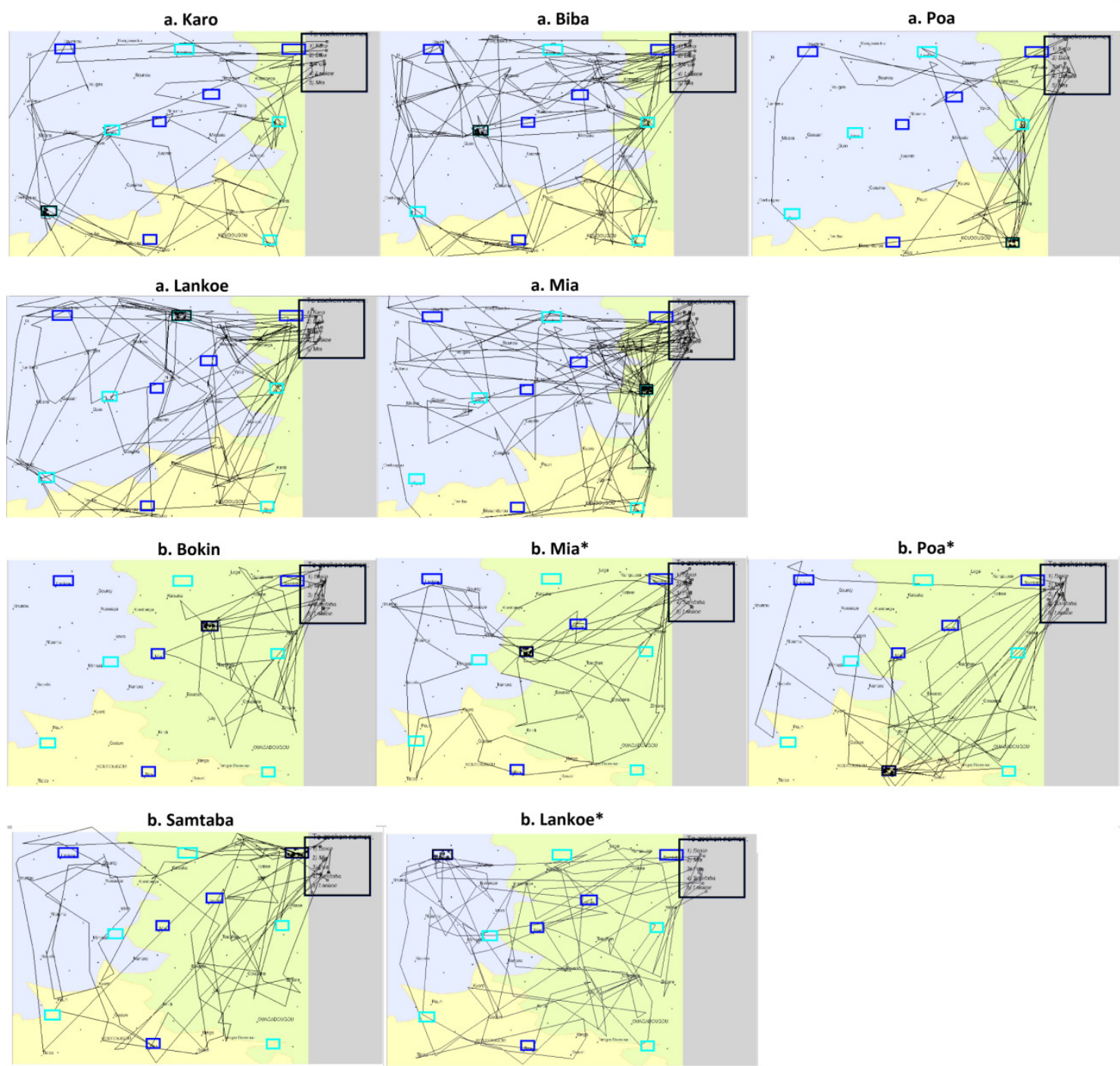

Figure 10: Division of the scanpaths to extract individual trajectories between visited areas 


\section{Conclusion}

The functions available in The Visual Analytics Toolkit include selection of the (eye) movement data based on attributes and/or time intervals, aggregation of the data based on a Voronoi tessellation or based on user defined areas, simplification of the data based on crossed (user defined) areas and subdivision of the trajectories (scanpaths) based on crossed areas. These techniques are applied to data in the main memory. However, the applicability of the approach is limited to about 20,000 - 50,000 scanpaths with up to 1,000,000 fixation points. Larger data sets require out-of-memory processing that integrates visual analytics with database sampling (G. Andrienko, et al., 2009).

The results obtained from the current study have given insights in how users process the information on a map when it is displayed for the first time and after an (simulated) interaction. Detecting patterns in the eye movement data is possible since the spatial dimension of the actual trajectories or scanpaths are visualised and analysed which is in contrast tot the conventional quantitative approach in the analysis of the eye tracking data. Pattern in this movement data such as the evolution of the search behaviour over time and the personal search strategies discussed above - cannot be detected when only analysing the measurements related to the fixations and saccades (e.g. duration and number).

\section{References}

Andrienko, G., Andrienko, N., Rinzivillo, S., Nanni, M., Pedreschi, D., \& Giannotti, F. (2009). Interactive Visual Clustering of Large Collections of Trajectories. In IEEE VAST 2009 (pp. 3-10). Atlanta Ciry, New Yersey, USA.

Andrienko, G., Andrienko, N., \& Wrobel, S. (2007). Visual analytics tools for analysis of movement data. SIGKDD explorations, 9, 38-46.

Andrienko, N., \& Andrienko, G. (2010). Spatial Generalization and Aggregation of Massive Movement Data. IEEE Transactions on visualization and computer graphics. http://doi.ieeecomputersociety.org/10.1109/TVCG.2010.44

Bevan, N. (1995). Usability is quality of use. Symbiosis of Human and Artifact: Human and Social Aspects of Human-Computer Interactions, 20, 349-354.

Brodersen, L., Andersen, J. H. K., \& Weber, S. (2001). Applying the eye-movement tradcking for the study of map perception and map design. In (pp. 98). Copenhagen, Denmark: National Survey and Cadastre.

Coltekin, A., Heil, B., Garlandini, S., \& Fabrikant, S. I. (2009). Evaluating the effectiveness of interactive map interface designs: a case study integrating usability metrics with eyemovement analysis. Cartography and Geographic Information Science, 36, 5-17.

Downs, R. M., \& Stea, D. (1977). Maps in Minds. Reflection on cognitive mapping. New York: Harper \& Row.

Duchowski, A. T. (2007). Eye movement analysis. In Eye tracking methodology - Theory and practice (pp. 137-156): Springer.

Fabrikant, S. I., Rebich-Hespanha, S., Andrienko, N., Andrienko, G., \& Montello, D. R. (2008). Novel method to measure inference affordance in static small-multiple map displays representing dynamic processes. The Cartographic Journal, 45, 201-215. 
ISO. (1994). ISO9241-11: Ergonomic requirements for office work with visual display terminals (VDTs). In Part11: Guidance on usability.

Jacob, R., \& Karn, K. (2003). Eye tracking in human-computer interaction and usability research: Ready to deliver the promises. In R. Radach, J. Hyona \& H. Deubel (Eds.), The Mind's Eye: Cognitive and Applied Aspects of Eye Movement Research (pp. 573-605). Amsterdan: Elsevier.

Montello, D. R. (2002). Cognitive map-design research in the twentieth century: theoretical and empirical approaches. Cartography and Geographic Information Science, 29, 283-304.

Nielsen, J. (1993). Usability Engineering. San Francisco: Morgan Kaufmann.

Poole, A., \& Ball, L. J. (2006). Eye tracking in human computer interaction and usability research: current status and future prospects. In C. Ghaoui (Ed.), Encyclopedia of Human Computer Interaction (pp. 211-219): Idea Group.

Rayner, K. (1998). Eye movement in reading and information processing: 20 years of research. Psychological Bulletin, 124, 372-422.

Rubin, J., \& Chisnell, D. (2008). Handbook of Usability Testing. How to Plan, Design and Conduct Effective Tests (second ed.). Indianapolis: Wiley Publishing. 